\title{
Comment on Tadrous et al.: Comparative gastrointestinal safety of bisphosphonates in primary osteoporosis: a network meta-analysis
}

\author{
M. Pazianas • B. Abrahamsen
}

Received: 6 May 2014 / Accepted: 19 June 2014 /Published online: 18 July 2014

(C) International Osteoporosis Foundation and National Osteoporosis Foundation 2014

Dear Editor,

The study by Tadrous et al. [1] identified zoledronic acid as the bisphosphonate with the "highest probability gastrointestinal (GI) adverse events (91\%) and nausea (70\%).” Many clinicians will have been surprised at this finding. First, in contrast to the established mechanisms of upper GI AE following oral preparation of bisphosphonates, there are no known mechanisms explaining GI AEs including nausea when bisphosphonates are administered in IV regimens. Of course, an acute face reaction is not uncommon after zoledronic acid infusion, especially after the first administration and more rarely after the next infusion and this could be accompanied in some cases by stomach upset and nausea. This could have been explored, had the authors included the acute face reaction in their analysis. Second, the frequency of events reported in Table 2 does not seem internally coherent. For

A reply to this comment can be found at DOI 10.1007/s00198-014-2789z.

M. Pazianas $(\bowtie)$

The Botnar Research Centre \& Nuffield Department of

Orthopaedics, Rheumatology \& Musculoskeletal Diseases, Nuffield

Orthopaedic Centre, Institute of Musculoskeletal Sciences, Oxford

University, Oxford OX3 7LD, UK

e-mail: Michael.Pazianas@ndorms.ox.ac.uk

B. Abrahamsen

Department of Medicine M, Glostrup Hospital, Glostrup, Denmark

B. Abrahamsen

OPEN, Institute of Clinical Research, University of Southern

Denmark, Odense, Denmark alendronate, $54 \%$ reportedly experienced esophageal AEs and $29 \%$ nausea, yet there was no discontinuation of alendronate due to GI adverse events and the total probability of any GI events was stated as $1 \%$. For zoledronic acid, the authors state that there was no reporting of specific outcomes such as upper GI events or esophageal events, yet cite a probability of any GI event of more than $90 \%$ with discontinuation in $14 \%$. At any rate, we are left with the impression that the included studies had extreme heterogeneity in their reporting of adverse events and that this may have led to a biased estimate favouring older studies. As a test of this, the authors could have compared the total AE event rate between the placebo groups in the trials. An unforeseen consequence of the conclusions of the study could be a shift away from zoledronic acid, which is now available as a cheaper generic preparation in many countries, to denosumab which remains on patent. Pending a more transparent and robust analysis, we are not persuaded that GI adverse events are more likely with zoledronic acid than with oral bisphosphonates. In reference to adherence, it is surprising the way the authors interpreted their findings when they concluded that annual administration of zoledronic acid may not improve adherence.

\section{Reference}

1. Tadrous M, Wong L, Mamdani MM, Juurlink DN, Krahn MD, Lévesque LE, Cadarette SM (2014) Comparative gastrointestinal safety of bisphosphonates in primary osteoporosis: a network metaanalysis. Osteoporosis Int 25(4):1225-35. doi:10.1007/s00198-013$2576-2$ 